La Rosa, G. (2020). Invito alla riscoperta e alla traduzione di Mario Rapisardi. Italica Wratislaviensia, 11(2), 137-160.

DOI: http://dx.doi.org/10.15804/IW.2020.11.2.8

\author{
Gabriele La Rosa \\ Uniwersytet Wrocławski, Polonia \\ gabriele.larosa@uwr.edu.pl \\ ORCID 0000-0002-7084-0895
}

\title{
INVITO ALLA RISCOPERTA \\ E ALLA TRADUZIONE DI MARIO RAPISARDI
}

\author{
AN INVITATION TO THE REDISCOVERY \\ AND TRANSLATION OF MARIO RAPISARDI
}

\begin{abstract}
This article is intended to constitute a first approach towards a critical, comparative, and bibliographic study of the forgotten figure of Catania-born Mario Rapisardi (1844-1912), "the Etnean poet." A poet and polemicist, he expressed himself in the climate of Positivism during and after Italian Unification, distinguishing himself with an ethical temper that did not make him abandon the Promethean tension towards ideals. Due to these ideals, the poet came into conflict with both the academic and ecclesiastical worlds, resulting in his consequent literary isolation - but not social and human isolation, clearly demonstrated by the fact that more than 100,000 people would attend his funeral.

This article is also an invitation to rediscover and translate Rapisardi's work into Polish, as his presence is almost non-existent, given that only four short poems are translated into Polish: three published in 1921 (Disinganno, La montagna fatale, Dinanzi a un ritratto) and one in 1931 (Nox). The translations are by Jadwiga Lipińska, Józef Puzyna, Julian Ejsmond, and Julia Dickstein-Wieleżyńska, respectively.

The article chronologically traces the Polish criticism of Rapisardi, starting from 1880 with two articles by Polish writers and literary critics Waleria Marrené-Morzkowska and Julian Adolf Święcicki, up to the present times, and is based both on studies by famous literary scholars, such as Maurycy Mann, and on articles that appeared in literary magazines, such as Przeglad Humanistyczny and Przegląd Wspótczesny.
\end{abstract}

Keywords: Rapisardi, Catania, Poland, translations, poetry 
Tl nome di Mario Rapisardi raramente trova spazio all'interno delle antologie scolastiche, tanto che il nome dell'illustre poeta catanese risulta pressoché sconosciuto ai più, sia in Italia sia all'estero. Incredibilmente, anche nella sua città natale si è perso il riferimento alla sua attività e del poeta rimane noto solamente l'omonimo lungo viale a lui intitolato, una delle arterie principali della città (Grasso, 2019, pp. 105140). I più addentrati nei fatti sono a conoscenza di un busto in bronzo ubicato nei giardini pubblici di Catania, la cosiddetta Villa Bellini, dedicata al musicista Vincenzo Bellini, che di contrapposto ha sempre goduto della massima popolarità. Sulla base del monumento ci sono due frasi, la prima di Victor Hugo: "Vous êtes un précurseur"1 e la seconda di Garibaldi: "Coraggio! All'avanguardia del progresso noi vi seguiremo" (Patanè, 1945, p. 68).

Questo studio vuole costituire un primo approccio verso un'indagine sulla dimenticata figura di Mario Rapisardi (1844-1912), "il vate etneo", come lui stesso si denomina nel suo poema L'Atlantide. L'articolo, dopo alcuni cenni biografici e di critica italiana, ripercorre cronologicamente la riflessione critica polacca su Rapisardi, dalla seconda metà del XIX secolo fino ai giorni nostri, basandosi sia sui contributi di importanti studiosi di letteratura come Maurycy Mann sia su articoli apparsi in riviste culturali, come Przeglad Tygodniowy, Przeglad Humanistyczny e Przeglad Wspótczesny. Il presente lavoro è anche un invito alla riscoperta e alla traduzione in polacco dell'opera del Catanese pressoché inesistente, visto che risultano tradotte in polacco solo quattro brevi poesie.

\section{CENNI BIOGRAFICI}

Nato da un'agiata famiglia borghese, figlio unico, Rapisardi trascorse la sua infanzia in piena libertà, senza venire mai richiamato o punito dai

1 L'iscrizione non riporta però le seguenti parole di Hugo: "Vous avez dans les mains deux flambeaux, le flambeau de poésie et le flambeau de vérité. Tout deux éclaireront 1'Avenir'. Come a volerlo ripagare, Rapisardi nello scritto di stampo teoretico La poesia filosofica saluta Hugo come il "più grande poeta [francese] del secolo". Lo scritto è del 2 marzo 1898 (Vigo-Fazio, 1930). 
genitori. Probabilmente, questa elusione delle figure di autorità plasmò il carattere baldanzoso del futuro versificatore e polemista.

La giovinezza di Mario fu segnata da problemi di salute: a diciotto anni si ammalò di emottisi, ovvero tossiva sputando sangue e dovette trascorrere sette difficili anni, nei quali si immerse in studi solitari e autonomi. Si iscrisse a giurisprudenza per seguire le orme del padre, procuratore legale, ma abbandonò l'università senza conseguire la laurea. Egli affermava di sé:

Di notevole non c'è nulla nella mia vita se non forse per questo, che, bene o male, mi sono formato da me, distruggendo la meschina e falsa istruzione ed educazione ricevuta, e istruendomi ed educandomi da me, a modo mio, fuori da qualunque scuola, estraneo a qualunque setta, sdegnoso di sistemi e pregiudizi. (Scuderi, 1968, p. 195)

I primi effetti di questo "studio matto e disperatissimo" furono la pubblicazione dei Canti (1863), titolo di sapore leopardiano². Nel 1865 visitò per la prima volta Firenze, la neocapitale del Regno d'Italia, meta di intellettuali e artisti, nella quale ritornerà spesso. Lì il giovane poeta entrò in contatto con l'ambiente culturale presso il quale introdusse Giovanni Verga e Luigi Capuana. In quegli anni conobbe Giovanni Prati, Niccolò Tommaseo e strinse amicizia con Francesco Dall'Ongaro, presso il quale fece la conoscenza di Giselda Fojanesi che sposò nel 1872.

Nel 1868 pubblicò il poema filosofico La Palingenesi, dove auspicava una "nuova nascita", ovvero un rinnovamento dello spirito religioso e della società umana. La tematica era più che attuale durante gli anni della "questione romana", e i propositi di armonia sociale tra Stato e Chiesa, e scienza e fede, non erano solamente una visione utopistica di Rapisardi, ma un importante campo di discussione ${ }^{3}$. Il successo de $L a$

${ }^{2}$ L'amore per il poeta di Recanati è riscontrabile anche nella variazione del cognome da Rapisarda a Rapisardi. Sebbene egli non abbia mai cambiato ufficialmente il cognome all'anagrafe, il Catanese ha utilizzato questa nuova desinenza per somigliare foneticamente a Leopardi. Ulteriore omaggio è la raccolta di poesie Le Ricordanze (1872).

3 Anche il Vaticano sarebbe entrato nell'argomento: papa Leone XIII nel 1891 pubblicò l'enciclica Rerum Novarum. 
Palingenesi gli diede notorietà e la città di Catania lo insignì di una medaglia d'oro che egli commentò così: "Era veramente tempo che le città italiane smettessero l'ingrata abitudine di onorare solamente gli estinti" (Giarrizzo, 1987, p. 8).

Gli anni successivi furono fertili per il poeta: nel 1870 fu chiamato a tenere un corso di letteratura italiana all'Università degli Studi di Catania, il che segnò l'inizio della sua carriera accademica che lo porterà a ricevere la nomina di professore straordinario. Nel 1877 pubblicò la sua opera più famosa, Il Lucifero, poema anticlericale che scatenò tante reazioni ${ }^{4}$ e diede scandalo al punto da essere censurato dall'arcivescovo di Catania. Lucifero è il protagonista ed è inteso in senso etimologico di 'portatore di luce' e progresso, rischiaratore delle menti umane grazie alla sua intelligenza, figlio di Prometeo. Nel bene e nel male il poema contribuì ad accrescere la notorietà di Rapisardi e l'anno seguente egli fu nominato ordinario di Letteratura italiana e di Letteratura latina, da parte di Francesco De Sanctis, suo estimatore e allora ministro della Pubblica Istruzione.

Rapisardi inviò in regalo una copia del poema a Giosuè Carducci, il quale si vide rispecchiato in alcuni versi non certo elogiativi, dando inizio alla lunga polemica col Catanese:

E chi in aspetto di plebeo tribuno

Giambi saetta avvelenati e cupi,

E fuor di sé non trova onesto alcuno:

Idrofobo cantor, vate da lupi,

Che di fiele briaco e di lièo,

Tien che al mio lato il miglior posto occùpi

(Opere di Mario Rapisardi, vol. 2, p. 272)

Quello che inizialmente era un semplice fraintendimento (più o meno voluto) assunse i toni di una vera e propria disputa che si protrasse a lungo, con un botta e risposta di articoli pubblicati su giornali e periodici

4 "Con feroce burla verso il Rapisardi" (Finocchiaro Chimirri, 1995, p. 21) venne pubblicato un libretto anonimo dal titolo Paralipomeni a Lucifero (1878) edito da Zanichelli. In seguito venne rivelato l'autore, Luigi Capuana. 
quali Fanfulla della Domenica, Capitan Fracassa, Stella d'Italia, Don Chisciotte, Cronaca Bizantina. Una sintesi di questa polemica si ebbe a ridosso di quegli stessi anni, nel celebre esordio letterario di Federico De Roberto, ovvero la Polemica Rapisardi-Carducci (1881). Lo scrittore napoletano riconosceva il valore di entrambi i poeti e auspicava un pronto estinguersi di questa sterile diatriba, ma non fu così: i toni si fecero sempre più accesi e le reciproche insofferenze personali presero il sopravvento, tanto che si arrivò al dileggio. E Carducci, apostrofato "lecchino" (Patanè, 1946, p. 97) rispose in tono razzistico al "Vil catanese" (ibid.) così: "I siciliani sono sopravvivenze di razze inferiori, soprattutto quando sono rapisardiani" (ibid., p. 98). Non occorre dilungarsi ulteriormente sulla ventennale polemica, ma vale la pena sottolineare l'eccezionalità del Rapisardi come polemista. Il critico Giovanni Falaschi precisa che il Siciliano non era un personaggio di secondo rango di cui si poteva facilmente aver ragione come, per esempio, era avvenuto con i due scrittori lombardi Bernardino Zendrini (1839-1879) e Giuseppe Guerzoni (1835-1886) (Falaschi, 1979, p. 23), anch'essi critici nei confronti di Carducci. E anche Cesare Testa, un amico intimo del Maremmano, scrive che Mario Rapisardi "è il solo poeta italiano contemporaneo che abbia strappato al Carducci un gesto mal represso, ancorché fugace, d'invidia" (ibid., pp. 100-101).

Nel 1883 la relazione con la moglie venne interrotta in seguito alla scoperta del suo tradimento con Verga e a questa profonda delusione amorosa, condita dall'inopportunità delle malelingue, si aggiunsero gli sforzi nel dover rispondere ai detrattori della sua opera come Corrado Ricci, Lorenzo Stecchetti e Luigi Lodi, che addirittura lo sfidò a duello (ma Rapisardi non raccolse la sfida). Ad essi egli rispose con il poema storico-filosofico Giobbe (1884), in cui è il personaggio biblico a rappresentare l'umanità intera. Anche questa opera venne bistrattata da Carducci e compagni, e questo contribuì ulteriormente ad annerire l'umore del Catanese, il quale trovò la propria àncora di salvezza nell'allora diciottenne Amelia Poniatowski Sabérnich. La giovane polacca di Firenze, conosciuta nel 1885, dapprima fu sua segretaria e in seguito sua fedele compagna che lo assistette amorevolmente per il resto della sua vita, ripagandolo delle sfortunate vicende sentimentali precedenti. 
Gli anni successivi portarono a Rapisardi nuove delusioni: dai problemi con la magistratura nel 1888 per la pubblicazione della poesia sociale Duetto alle accuse da parte dei socialisti estremisti, i quali rinfacciavano al Catanese di aver tenuto un profilo basso durante la repressione dei Fasci Siciliani del 1893/18945. Nello stesso anno pubblicò il poema di matrice socialista L'Atlantide, anch'esso non apprezzato dalla critica. Nel 1898 in occasione del trentennale della pubblicazione de La Palingenesi, vennero organizzate solenni onoranze per il poeta e professore Rapisardi ${ }^{6}$. L'iniziativa fu presa da un gruppo di studenti universitari e pian piano coinvolse gli altri due atenei siciliani (Palermo e Messina) per poi estendersi a tutta Italia. Molte università italiane ed estere espressero la loro adesione e venne costituito un comitato al quale si aggregarono intellettuali di tutta Italia e stranieri. Fra questi si ricordano: i letterati Luigi Capuana, Felice Cavallotti, Edmondo De Amicis, Federico De Roberto, Arturo Graf, Giovanni Pascoli, Émile Zola, Jules Destrée, il linguista Graziadio Ascoli, l'antropologo Cesare Lombroso, lo psichiatra Enrico Morselli e il biologo Ernst Haeckel. Gli atti di tali onoranze vennero pubblicati l'anno seguente (Campanozzi, 1899) e nello stesso anno venne esposto il busto bronzeo del poeta, testimonianza dell'amore che i suoi concittadini avevano per lui, soprattutto gli studenti che seguivano con viva passione i suoi insegnamenti cattedratici. Lui stesso descrisse la sua attività didattica con queste parole:

Nelle mie lezioni, studio di preferenza il carattere morale dei grandi scrittori, giacché son persuaso che lo studio delle lettere è studio d'umanità, che la scuola è una palestra morale, una preparazione alle battaglie della vita. Faccio critica scientifica piuttosto che letteraria, e se ho per oggetto principale la letteratura italiana, non trascuro lo studio dei problemi estetici e morali che vi si connettono, né le necessarie comparazioni con le letterature contemporanee. (Manganaro, 2010, p. 57)

5 Per approfondimenti si veda la lettera del 10 febbraio 1894, pubblicata come prefazione al volume di Napoleone Colajanni, Avvenimenti di Sicilia e le loro cause (1894).

6 Va ricordato che Rapisardi fu attivo anche come traduttore dal latino (Catullo, Lucrezio, Orazio, Virgilio, Boezio) e dall'inglese (Thomas Moore e Percy Bysshe Shelley). 
Nonostante questi apprezzamenti, negli ultimi anni, il poeta si chiuse in sé e il suo carattere iracondo, poco avvezzo alle critiche e alla diplomazia, non lo aiutò sicuramente. Ne è esempio il suo particolare biglietto da visita che riportava le seguenti parole:

Mario Rapisardi non iscrive nei giornali; non accetta nomine accademiche. Né candidature politiche ed amministrative; non vuol essere aggregato a nessun sodalizio; non ha tempo di leggere tutti i libri che gli mandano, molto meno i manoscritti; né di rispondere a tutti coloro che gli scrivono. E di ciò chiede venia a discreti. (La Vecchia, 2001, p. 61)

Anche a causa delle condizioni psicofisiche precarie, egli si isolò sempre più vivendo gli ultimi anni come un eremita e alimentando così il proprio mito. Il suo animo inquieto visse però placato grazie all'appiglio umano di Amelia Poniatowski Sabérnich, "bella e colta discendente di sovrani polacchi $[\ldots]$ la giovane che tutto il suo spirito consacrò a Rapisardi: l'ispiratrice, l'infermiera, la consolatrice dell'«arcangelo fulminato»" (Patanè, 1946, pp. 67 e 94).

Non amato particolarmente dalla critica, ma non per questo non ammirato dal popolo, affascinato dai suoi slanci prometeici a favore delle masse, il poeta si spense il 4 gennaio 1912. La città tenne il lutto per tre giorni e al suo solenne funerale parteciparono oltre centomila persone vestite a festa, il feretro con il corpo imbalsamato ${ }^{7}$ del poeta sfilò per le vie di Catania nella storica carrozza del senato civico, e il cordoglio dei concittadini si trasformò in partecipazione giocosa "come se il funerale fosse stato la celebrazione del centenario di un santo" (ibid., p. 138).

\section{CRITICA ITALIANA}

Il Rapisardi fu un apprezzato scrittore sin da giovane, tanto che già nel 1872 venne pubblicato uno studio critico-letterario firmato dal catanese Nicola Niceforo e stampato per la prima volta nella Nuova Enciclope-

\footnotetext{
7 Per quanto riguarda le vicende dell'imbalsamazione e del funerale si veda Centorbi (1975). Per quanto riguarda invece le bizzarre vicende della difficile e lunga tumulazione del corpo si veda Vigo-Fazio (1913).
} 
dia Italica di Roma; nel breve saggio Rapisardi è definito una "delle più complete manifestazioni del sentimento artistico italiano" (Niceforo, 1872 , p. 38). Circa il valore poetico della sua opera, durante la sua vita si assistette ad una polarizzazione tra estimatori e detrattori, questi ultimi incoraggiati dalla polemica col Carducci. Rapisardi era circondato e quasi idolatrato da una cerchia di discepoli, intellettuali, artisti e ammiratori quasi tutti catanesi: "Attorno alla figura zazzeruta di Mario Rapisardi $[\ldots]$, si era formato un vero e proprio mito del genio poetico" (Finocchiaro Chimirri, 1995, p. 21). Alcuni di essi addirittura lo imitavano anche nell'aspetto, portando appunto la zazzera, come il pittore Calcedonio Reina, il poeta Gaetano Ardizzoni e il discepolo Alfio Tomaselli ${ }^{8}$.

Fra gli altri artisti che si ricordano c'erano il pittore Antonino Gandolfo (autore del celebre ritratto del Poeta), l'incisore Francesco Di Bartolo, il musicista Francesco Paolo Frontini. I seguaci del Poeta si riunivano nella bottega di un calzolaio, don Alfio Scandurra, uno dei suoi maggiori estimatori; in una bella bottega in via Etnea e non in salotti aristocratici o mondani da cui il Poeta si teneva ben lontano, coerentemente con il suo orizzonte ideologico. C'era infine la moltitudine dei cittadini che osannavano il Poeta pur non conoscendo la sua opera. Il popolo era principalmente attratto dal suo anticlericalismo e dai suoi ideali socialisteggianti. Rapisardi era mal visto dalla nobiltà, ma poteva contare su appoggi di personaggi illustri e influenti, come il deputato socialista Giuseppe De Felice Giuffrida, sostenitore dei Fasci, il quale stimava il Poeta per via del suo atteggiamento in favore del popolo di contadini e minatori, sfruttato da "proprietari e gabellotti [che] avevano accumulato grandi ricchezze" (Sciascia, 1970).

Fra le cause che hanno contribuito al prolungato oblìo del poeta, oltre alla sterile polemica con Carducci, pesò anche il giudizio di Benedetto Croce, sostanzialmente negativo, secondo il quale si dovrebbero "gittare all'onda letea i nove decimi, più e non meno, della roba che egli ha scritto, carezzato, ristampato, e che ha lasciato ammirare dai suoi fa-

8 Amico intimo di Rapisardi, dopo la morte di questi, sposò Amelia e curò i carteggi, le opere postume e pubblicò il voluminoso Commentario rapisardiano (1932). 
natici, e sulla quale si fonda principalmente la sua fama o la sua notorietà" (Croce, 1905, p. 90). Il critico ribadisce di non apprezzare Rapisardi, né come autore, per via delle "bruttezze dei suoi poemi" (ibid., p. 97) né come tribuno quando si erge cantore delle masse oppresse. Croce ha una preclusione per l'arte di ispirazione socialista, egli boccia la raccolta di poesie sociali Giustizia (1883), di cui salva solo il Canto dei mietitori. Fra le altre opere apprezzate da Croce, pregne di una sincera commozione poetica in cui prevale Rapisardi come artista e non come retore, c'è il poema L'Empedocle (1892) e la poesia Mors et Vita (1895), un componimento poetico che il critico Giuseppe Nicolosi definisce "un capolavoro sia per vigoria di stile, che per vivente bellezza d'immagini, e per profondità di pensiero" (Nicolosi, 1921, p. 2). Anche Luigi Russo in una delle sue prose polemiche dal titolo Il tramonto del letterato (1920), parlando dell'orizzonte culturale italiano, auspica che non ci sia: "l'avvento di apostoli siano essi filosofi o poeti, che invasati di furore patriottico, sociale o rivoluzionario, si facciano banditori di verbi astratti di vita, nelle loro filosofie e nelle loro poesie" (Falaschi, 1979, p. 73). Russo ha certi pregiudizi sugli aspetti non puramente letterari e per questo considera Rapisardi, insieme a Vincenzo Monti e Giovanni Prati, "ultimi epigoni del tomismo estetico, costruttori di cattedrali, in un'età in cui il pur giovane dio cristiano ne era esulato" (Russo, 1967, p. 161). Il giudizio di Gramsci invece è più neutrale. Egli sembra quasi interessato a vagliare l'ascrivibilità di Rapisardi al materialismo storico: "È ciò vero? O non piuttosto fu egli un «mistico» del naturalismo e del panteismo? Però legato al popolo, specialmente al popolo siciliano, alle miserie del contadino siciliano ecc." E un'opera come Giustizia è per il critico "poesia da democratico-contadino". Gramsci, più attento a porre domande che non a fornire risposte, segnala l'ottimo saggio del critico ennese Nunzio Vaccalluzzo come un buon punto di partenza "per iniziare uno studio sul Rapisardi" (Gramsci, 1948-51, Quaderno VI, 57). Vaccalluzzo ricorda che dopo la morte del poeta ebbe inizio una revisione critica della sua arte e non mancarono coloro che lo apprezzarono, come ad esempio Attilio Momigliano, Giuseppe Antonio Borgese, Arturo Graf, il latinista napoletano Carlo Pascal, l'anticrociano Alfredo Galletti e Arnaldo Alterocca. Tutti questi critici concordano sul 
valore poetico del poema Il Giobbe, liquidato sommariamente da Croce come "più decoroso [degli altri, ma] affatto vuoto" (Croce, 1905, p. 93). A questi nomi si aggiunge anche Giacomo Samperisi (1922), autore di una voluminosa monografia sulla poesia di Rapisardi, scritta per il decennale della morte.

Durante il ventennio fascista, Rapisardi venne sempre più dimenticato, a causa dei contenuti anticlericali della sua opera, in contrasto con la dominante linea cattolica nonché per le sue posizioni socialiste e materialistiche. Pertanto, il giudizio di Vaccalluzzo, espresso nel 1930, rimane ancora molto attuale:

La vena genuina della poesia del Rapisardi è stata dunque intravista da critici autorevoli; e non dovrebbe perciò esser più possibile indugiare in lunghe analisi demolitrici de' poemi più scadenti e finger d'ignorare il Giobbe e le liriche posteriori o alla loro bellezza accennare, distrattamente e freddamente. (Vaccalluzzo, 1930)

$\mathrm{Nel}$ secondo dopoguerra inizia, seppur non energicamente, la rivalutazione dell'opera del vate etneo. Alberto Asor Rosa, ad esempio, accosta Mario Rapisardi a Olindo Guerrini (1845-1916), il poeta di Forlì noto con lo pseudonimo di Lorenzo Stecchetti'. In entrambi c'è l'interesse per gli aspetti drammatici della vita dei miseri: i "motivi della fame, dell'asservimento, della ribellione popolare cieca e crudele" (Asor Rosa, 1966, p. 53). Guerrini descriveva la realtà dei lavoratori nei campi della Bassa emiliana, Rapisardi invece i contadini siciliani, ma entrambi constatano che "per le masse popolari e contadine le imprese risorgimentali non hanno rappresentato nessuna sostanziale conquista" (Ivi, p. 54). Sono le tematiche della letteratura progressista: la fiducia nella scienza e nel progresso, il laicismo, l'anticlericalismo, l'irrisione dei dogmi e la lotta all'analfabetismo. Analogamente, il critico Salvatore Guglielmino annovera Rapisardi fra i poeti del periodo positivistico che hanno celebrato la scienza: accanto ad autori maggiori quali "Carducci che celebrò il treno come emblema del progresso" (Guglielmino, 1978, p. 13), ci sono autori minori come Giovanni Bertacchi (1869-1942)

9 Si ricordi che egli fu uno dei "paladini” del Carducci nella discussa polemica. 
e Rapisardi, il quale, nel poema Il Lucifero, professò la propria fede nel progresso.

Il 1987 diede occasione di commemorare il $75^{\circ}$ anniversario $^{10}$ della scomparsa di Rapisardi. Lo storico Giuseppe Giarrizzo, preside della Facoltà di Lettere e Filosofia dell'Università di Catania, fece il punto della situazione sul caso Rapisardi chiedendosi:

Può il lento prevalere di un giudizio storico, più equo e partecipe, di quel lontano periodo e del positivismo e della "poesia civile", già avviato con esito problematico per la scapigliatura e la borghesia lombarda, estendersi alla letteratura del secondo '800 meridionale e al Rapisardi? (Giarrizzo, 1987, p. 25)

L'intervento di Giarrizzo purtroppo non ha trovato grande eco in ambito accademico ${ }^{11}$, probabilmente perché avvenuta in occasione di un evento locale come il XVII Premio Letterario "Brancati-Zafferana", e non in un ambiente di maggior respiro. Sebbene alcuni scrittori si siano mossi, come i catanesi Carmelo Ciccia (1997), Sebastiano Barbagallo (1999), Francesco Giordano $(1998,2007,2011)$ e il genovese Virgilio Zanolla (1993 e 2011), Rapisardi rimane circoscritto all'ambiente culturale siciliano o meglio catanese ${ }^{12}$, mentre è del tutto sconosciuto al grande pubblico anche a causa della produzione in versi di difficile fruizione da parte di un lettore medio, soprattutto al giorno d'oggi. A detta di ciò appare abbastanza grave il fatto che nel 2012, per il centesimo anniversario della scomparsa dell'autore, il comune di Catania non abbia organizzato nulla per commemorarlo e per ricordare che Mario Rapisardi è "una persona prima che un viale" (Santangelo, 2012).

$10 \mathrm{Si}$ veda la pubblicazione I poeti e le poesie in onore di Mario Rapisardi nel $75^{\circ}$ anniversario della scomparsa, a cura di Michele D'Agata.

11 Ad esempio, Enzo Noè Girardi nella sua Letteratura italiana e religione negli ultimi due secoli (2008), nonostante l'argomento trattato, non accenna minimamente a Rapisardi.

12 La figura della moglie Giselda Fojanesi (e il triangolo amoroso con Verga e Rapisardi) ha trovato in questi ultimi anni due trasposizioni narrative nelle opere degli scrittori catanesi Piero Isgrò (La sposa del nord, 2014) ed Emanuela E. Abbadessa (Fiammetta, 2016). 


\section{CRITICA POLACCA}

La prima testimonianza su Rapisardi appare nel 1880 con la scrittrice e critica letteraria polacca Waleria Marrené-Morzkowska, la quale pubblica un articolo ${ }^{13}$ sullo scrittore Roman Zmorski, sul Przeglad Tygodniowy Życia Społecznego, Literatury i Sztuk Pięknych ${ }^{14}$. La Marrené-Morzkowska sottolinea come Zmorski tratti la figura di Satana, nella poesia Noc świętojańska (1842) e soprattutto nel romanzo in versi Lesław. Szkic fantastyczny (1847). La scrittrice ripercorre rapidamente il mito di Satana, trovando esempi nel poema Il paradiso perduto (1667) di John Milton e nel poema drammatico Manfred (1817) di Lord Byron, e ricorda che anche nella letteratura italiana contemporanea ci sono autori che hanno trattato lo stesso tema, come Carducci con l'Inno a Satana (1863) e Rapisardi con Il Lucifero. Secondo lei, entrambe le opere assumono il principe delle tenebre come rappresentante della Ragione, che si erge contro Dio. Pertanto, Zmorski è accomunabile a Rapisardi per via degli aspetti mistici, anche se nell'opera del polacco mancano le idee del clima culturale positivistico ancora a venire.

Qualche mese dopo lo scrittore e traduttore Julian Adolf Święcicki firma un capitolo, dedicato alla letteratura italiana contemporanea, nel secondo volume della Biblioteka Warszawska (1880). Il saggio, ben costruito e corredato di un valido apparato bibliografico, annovera il Siciliano fra i giovani poeti, tratteggiando brevemente il suo pensiero, che si muove dalla fede al dubbio per poi approdare ad

13 L'articolo Grupa poetów z 1840 roku, pubblicato in quattro numeri della rivista, è stato oggetto di studio da parte di Halina Krukowska e Jarosław Ławski, i quali lo hanno riproposto nel volume su Roman Zmorski (1822-1867) dedicato all'opera Lesław. Il volume riporta anche la copertina de Il Lucifero (p. 8), contenente un'illustrazione a colori firmata da Gino De Bini (1887). Il curatore del volume Ławski però si è limitato a riprendere il punto di vista della Marrené-Morzkowska tanto che egli riporta lo stesso errore della polacca, la quale afferma che Rapisardi è "un poeta di Napoli" (Krukowska \& Lawski, 2014, p. 162).

14 Settimanale di vita sociale, letterature e belle arti, edito a Varsavia negli anni 1866-1904. Nelle sue pagine trovarono spazio autori della corrente positivistica. Il redattore era il giornalista Adam Wiślicki, curatore della Podręczna encyklopedia powszechna (1873-1901). 
un pieno scetticismo. Święcicki presenta molto sinteticamente tre sue opere: La Palingenesi "che delinea una storia del pensiero cristiano", Le Ricordanze "che riproduce la lotta interiore dell'anima" e Il Lucifero "in cui impersona il pensiero umano in tutta la sua grandezza e miseria". La conclusione finale di Święcicki è che "non si può essere d'accordo con Rapisardi come filosofo, ma bisogna amarlo come poeta" (Święcicki, 1880, p. 372).

Nel 1901 Zenon Przesmycki, redattore della rivista Chimera $^{15}$, firma (con lo pseudonimo Miriam) l'articolo Poezja, nel quale commenta le traduzioni di due opere di Ada Negri, da parte di Maria Konopnicka, affermando che l'editore avrebbe potuto sfruttare meglio il talento della celebre traduttrice, attingendo da buoni poeti italiani quali Carducci, Rapisardi, D'Annunzio e Pascoli.

Nel 1902 l'italo-polacca Maria Rygier ${ }^{16}$ firma un lungo articolo sulla letteratura italiana contemporanea apparso su tre numeri del Tygodnik Stowa Polskiego ${ }^{17}$ di Leopoli. Ella ribadisce l'importante ruolo di Carducci, ma ricorda che egli non è il solo. Accanto al Maremmano c'è tutto un gruppo di poeti nati tra il 1830 e il 1845 che guardarono con particolare interesse ai precedenti scrittori impegnati su temi patriottici (Manzoni, Leopardi, Monti, Foscolo). Di questa generazione fanno parte Enrico Panzacchi, Giuseppe Aurelio Costanzo, Mario Rapisardi e Olindo Guerrini, mentre il gruppo successivo è quella dei nati dopo il 1850 (Pascoli, D'Annunzio etc.). Il Catanese è particolarmente originale perché è "romantico nei contenuti, ma classico nella forma" (Rygier,

15 Rivista letterario-artistica edita a Varsavia negli anni 1901-1907. Il redattore era Zenon Przesmycki (1861-1944), poeta, critico e traduttore da diverse lingue (dall'italiano tradusse Carducci). Fu uno dei rappresentanti della corrente modernistica Młoda Polska.

16 Nata a Cracovia nel 1885, figlia dello scultore polacco Teodor, autore del celebre monumento di Adam Mickiewicz a Cracovia. Da giovane si interessò di letteratura, poi intraprese la carriera politica in Italia. Al tempo in cui scrisse l'articolo non aveva compiuto nemmeno diciassette anni.

17 Il quotidiano Słowo Polskie fu edito a Leopoli dal 1896 al 1934. Negli anni 19021903 venne pubblicato un suo supplemento settimanale, il Tygodnik Stowa Polskiego. Il redattore era Jan Kasprowicz (1860-1926), poeta, critico, drammaturgo e traduttore da diverse lingue (dall'italiano tradusse D’Annunzio), esponente della Młoda Polska. 
1902, p. 3). Egli è "dotato di una grande immaginazione poetica e di uno stato d'animo allegro; trova però pochi seguaci, il migliore dei quali è Alfredo Cesareo" (ibid.), poeta, critico e drammaturgo siciliano.

Nel 1904 Antoni Lange ${ }^{18}$ firma (con lo pseudonimo di A. Wrzesień) un articolo sulla letteratura italiana contemporanea sul settimanale illustrato per donne Bluszcz ${ }^{19}$, nel quale tratta di Cavallotti, Costanzo e Rapisardi, "l'autore del grande poema in lode di Satana (Lucifero)" (Lange, 1904, p. 357). Nel 1909 Józef Sebastian Pelczar, vescovo di Przemyśl, pubblica un volume sulla massoneria e cita in nota Rapisardi annoverandolo fra gli autori che "con enfasi hanno scritto di Satana" (Pelczar, 1909, p. 47).

Nel primo dopoguerra vedono la luce le traduzioni in polacco di quattro poesie di Rapisardi. Tre di esse nel 1921: Disinganno ${ }^{20} /$ Rozcza- $^{-}$ rowanie di Jadwiga Lipińska ${ }^{21}$ e La montagna fatale ${ }^{22}$ / Góra fatalna, di Józef Puzyna ${ }^{23}$, apparse nel volume dedicato all'Italia in Panteon literatury wszechświatowej (1921); Dinanzi a un ritratto ${ }^{24} /$ Sonet $^{25}$ del giovane poeta Julian Ejsmond (1892-1930) sul Kurier Polski. La quarta

18 Intellettuale poliedrico di Varsavia (1863-1929) ed esponente della Młoda Polska, fu poeta, filosofo, critico, giornalista e traduttore. Celebre poliglotta, dall'italiano tradusse Pico Della Mirandola e Gianbattista Vico.

19 Settimanale illustrato per donne fondato da Michał Glücksberg ed edito a Varsavia negli anni 1865-1939.

${ }^{20}$ Pubblicata ne Le ricordanze (1873), ora in Opere di Mario Rapisardi, Vol. I., p. 491.

21 Jadwiga Lipińska, poetessa, autrice della raccolta di poesie Kwiaty maku (1914). Nel volume con la traduzione di Rapisardi figurano anche altri poeti tradotti come E. Carmelo, G. Finzi e G. Pascoli.

${ }_{22}$ Pubblicata in Empedocle ed altri versi (1892), ora in Opere di Mario Rapisardi, Vol. V, p. 293.

23 Józef Edward Puzyna (1878-1949), storico e poeta. Nello stesso volume sono presenti anche traduzioni dei poeti E. Panzacchi, A. Graf, G. Pascoli, D. Angeli, E. A. Butti e A. Costanzo.

${ }^{24}$ Pubblicato ne Le ricordanze (1873), ora in Opere di Mario Rapisardi, Vol. I., pp. 486-487.

${ }^{25}$ Questa poesia non è inclusa nel volume sulle traduzioni dei poeti italiani da parte di quattro italianiste di Cracovia (Miszalska,Gurgul, Surma-Gawłowska, \& Woźniak, 2007). 
poesia $\mathrm{Nox}^{26} / \mathrm{Nox}^{27}$, della Julia Dickstein-Wieleżyńska (1881-1943), fu pubblicata sulla rivista Przeglad Humanistyczny ${ }^{28}$ (1931). Per tutte e quattro le poesie non sono indicati i titoli originali ma solo quelli tradotti in polacco; inoltre non è presente nessun commento o nota.

Nel 1930 Stanisław Wędkiewicz, professore dell'Università Jagiellonica, firma un lungo articolo sul Przeglad Współczesny (rivista da lui diretta) che tratta del fascismo e della cultura intellettuale dell'Italia nel dopoguerra. Egli accenna al Catanese, annoverandolo tra gli intellettuali socialisti insieme a Edmondo De Amicis e Ada Negri (Wędkiewicz, 1930, p. 315).

Nel 1933 vedono la luce due pubblicazioni sulla letteratura italiana, presenti in volumi collettanei a cura di Stanisław Lam. La prima è il Panteon wielkich twórców poezji i prozy, nelle cui pagine dedicate all'Italia a cura di Edward Porębowicz, Rapisardi non è incluso tra gli autori antologizzati, mentre risultano presenti Carducci e D'Annunzio. La seconda pubblicazione è Wielka literatura powszechna, dove è presente un saggio di Maurycy Mann, professore dell'Università di Varsavia (1920-1932). Egli vede in Rapisardi un poeta filosofo, una figura simile a quella del francese Sully Prudhomme (1839-1907), e identifica nella poetica del Siciliano due fonti: la filosofia naturale, il materialismo di Lucrezio (del quale il Catanese ha tradotto De rerum natura nel 1880) e la dottrina del socialismo. Inoltre, Lam accosta Rapisardi a Vincenzo Monti quanto a stile e cita le sue opere principali, ovvero La Palingenesi, Il Lucifero, Il Giobbe, Giustizia e L'Atlantide, nelle quali Rapisardi ammirava la natura e il pensiero umano libero da superstizioni religiose e morali, sognava una nuova religione e difendeva il socialismo. Mann, analogamente al critico italiano Russo, afferma che "Rapisardi ricorda

26 Pubblicata in Le poesie religiose (1895), ora in Opere di Mario Rapisardi, Vol. IV, Giannotta, pp. 470-471.

27 Di questa poesia la bibliografia Bara (http://bar.ibl.waw.pl/) registra anche un altro titolo, Nos. Sicuramente è un errore perché il titolo si riferisce alla parola latina nox cioè 'notte' e quindi in polacco noc e non nos che significa 'naso'.

28 Rivista trimestrale di scienza e pedagogia edita a Varsavia negli anni 1922-1933. Si occupava anche di filologia e letteratura e era diretta dallo storico della filosofia Wiktor Wąsik e dal filologo Stefan Cybulski. 
Monti, col quale aveva molto in comune" (Mann, 1933, p. 240) e accenna alla polemica con Carducci e al fatto che il Siciliano abbia avuto tanti ammiratori quanti detrattori. Infine, scrive che della sua vasta produzione ci sono opere le quali meriterebbero di essere ricordate, come ad esempio Mors et Vita.

Nella seconda metà del Novecento il poeta scompare quasi del tutto. Nella traduzione in polacco del volume di Natalino Sapegno (1969, $1^{\text {a }}$ edizione italiana 1948) il poeta è citato solamente in nota ed è accostato al poeta veneto Giacomo Zanella (1820-1888) per via della loro attività in "un ambiente periferico e provinciale ai margini della cultura viva; entrambi i poeti sentono l'influenza delle idee contemporanee, ma solo per farne un pretesto per esercizi di eloquenza letteraria, elegante e discreta in Zanella, grandiosa e sonora in Rapisardi" (Sapegno, 1969, p. 616). Nei manuali polacchi di storia della letteratura italiana, Joanna Ugniewska (1985 e 2001), Józef Heistein (1987), Piotr Salwa (1997), Krzysztof Żaboklicki (2008), nonché nell'opera collettanea a cura di Anna Tylusińska-Kowalska dedicata alla letteratura del paesaggio della Sicilia (2011), l'autore è assente. Qualche accenno invece si trova in Monika Gurgul che accomuna Rapisardi a Guerrini, poeti non della dimensione di Leopardi, ma che vale la pena studiare per via delle loro proposte poetiche (Gurgul, 2006).

$\mathrm{Si}$ conclude questo excursus sulla critica polacca con Wojciech $\mathrm{Gu}-$ towski, autore di una monografia (2001) sui motivi cristiani nella letteratura della Młoda Polska. Lo studioso accenna in nota a Rapisardi limitandosi a ricordare la rivalità col Carducci e la paternità del poema Il Lucifero. Un simile interesse religioso per Rapisardi emerge anche dal recente articolo (2017), apparso sulla rivista Polonia Christiana, del frate francescano di Wrocław, Wawrzyniec Maria Waszkiewicz, che cita Il Lucifero come esempio di letteratura massonica italiana.

Riassumendo, la critica polacca iniziò ad interessarsi di Rapisardi nel 1880 , poco prima che la polemica ${ }^{29}$ col Carducci degenerasse e aves-

29 Anche se Carducci fece notare la propria disapprovazione già nella lettera del 19 febbraio 1877 (Tomaselli, 1932, p. 141), la polemica toccò l'apice nel 1881. I critici polacchi quindi probabilmente ancora non ne erano a conoscenza. 
se eco sulle riviste. Il commento di Święcicki quindi si basa solo sulle opere (che potrebbe aver letto in italiano) La Palingenesi, Le ricordanze e Il Lucifero, che egli apprezza per la loro natura poetica ma non per quella filosofica. L'interesse è quindi puramente per il valore artistico di Rapisardi, mentre nel commento della Marrené-Morzkowska prevalgono gli aspetti contenutistici che fanno de Il Lucifero un'opera della civilizzazione secondo la visione del filosofo inglese Henry Thomas Buckle, uno degli ispiratori del pensiero polacco positivistico. Rapisardi è quindi visto come poeta e come filosofo, ma in ogni caso dalla lettura delle fonti non si ha affatto l'impressione che il Siciliano sia considerato un autore minore, tanto che il poeta e traduttore Przesmycki lo indica fra i buoni poeti italiani dai quali si dovrebbe attingere, senza ulteriori distinzioni di valore tra Carducci e i successivi Pascoli e D'Annunzio. Anche la Rygier, pur riconoscendo l'autorevolezza di Carducci, non sminuisce i restanti poeti che appartengono alla stessa generazione. L'unica critica che sminuisce il valore del poeta è quella di Mann: il suo articolo ricorda la critica di Croce, anche se quest'ultimo non appare direttamente nelle fonti citate ${ }^{30}$. Mann infatti inizia l'articolo ricordando gli autori che stanno alla base del pensiero di Rapisardi, cita le stesse opere che cita Croce e conclude dicendo che vale la pena salvare solamente qualche pagina, "soprattutto le piccole opere liriche, non prive di toni sublimi, e il poema lirico riflessivo Mors et Vita" (Mann, 1933, p. 240).

Si segnala infine che la critica polacca non si è soffermata su alcuni evidenti riferimenti alla Polonia da parte di Rapisardi. Eppure il poeta ha dedicato dei versi alla nazione amica, pochi ma di intenso significato. A diciannove anni, nel maggio 1863 il poeta scrisse l'ode Alla Polonia, pubblicata l'anno successivo nelle Odi civili. Cenni su questa ode sono presenti nell'articolo di Rita Verdirame pubblicato su Kwartalnik Neofilologiczny. La docente dell'Università di Catania definisce l'ode “ambi-

30 Nella bibliografia riportata da Mann sono presenti: la voluminosa Storia letteraria d'Italia, scritta da una società di professori (1926); A. Bartoli, Storia della letteratura italiana (1878-1889); V. Rossi, Storia della letteratura italiana (1921); F. De Sanctis, Storia della letteratura italiana (1870). 
ziosa nell'assetto argomentativo (e ampollosa, secondo l'impianto formale prediletto dall'autore), che riassume esemplarmente i paradigmi e gli stilemi adottati dalla vulgata rimeria patriottica italiana e sovranazionale" (Verdirame, 2012, p. 179).

\section{CONCLUSIONI}

Non esiste un vero e proprio studio critico polacco su Mario Rapisardi. La critica è davvero esigua e cita il poeta quasi sempre in nota o en passant per via della fama del suo Lucifero, senza aggiungere nessuna analisi approfondita; pertanto non ci sono i presupposti per poter parlare di fortuna/sfortuna di Rapisardi. Gli articoli degli studiosi di letteratura italiana, più lunghi, sono quelli di Święcicki e Mann, e constano rispettivamente di 11 e 20 righe. Il primo articolo loda il Catanese, il secondo invece sembra riportare i pregiudizi di Croce; e per questo le osservazioni di Mann andrebbero prese con le dovute riserve. Egli afferma che la notorietà di Rapisardi "è finita con la sua vita" (ibid.). In realtà ciò non è vero, infatti il requiescat per il poeta tardò un po' e nel primo numero della rivista catanese Pickwick (10 marzo 1915) il suo nome è, per esempio, ancora richiamato negli ironici versi delle pagine programmatiche: "Diffondete PICKWICK / 1' unico giornale siciliano che lasci dormire in pace / Mario Rapisardi e che rinunzi ad occuparsi dello / annoso problema meridionale" (Finocchiaro Chimirri, 1995, p. 21). Mann scrive l'articolo nel 1933 e non sembra tener conto di nessuno studio critico su Rapisardi apparso in Italia nel primo dopoguerra. Inoltre, per poter formulare un giudizio di questo tipo Mann avrebbe dovuto leggere le centinaia di pagine della vasta produzione del Siciliano e se lo avesse fatto probabilmente avrebbe speso più di mezza pagina per trattare il complesso Poeta.

La critica polacca presenta una visione del Poeta meno variegata di quella italiana, difatti non accenna alle vicissitudini della polemica col Carducci e alla sua vena di polemista. Per quanto riguarda le idee del Poeta, solamente Święcicki e Mann lo etichettano (pur non apprezzandolo) come filosofo per via delle sue idee positivistiche. Secondo Rapisardi, la fede nel progresso si rivela positiva per l'umanità ma negativa 
per gli uomini, da qui il connubio dell'atteggiamento titanico e pessimistico che contribuiscono a rendere il Siciliano un personaggio di difficile comprensione. I restanti critici polacchi si limitano ad etichettarlo come intellettuale socialista (Wędkiewicz) o semplicemente come colui che ha trattato di Satana (Marrené-Morzkowska, Lange, Pelczar, Gutowski, Waszkiewicz). Infine, per quanto riguarda le opere, la critica italiana elogia soprattutto il poema Il Giobbe, assente nella critica polacca e appena citato in quella di Mann.

All'interno del panorama letterario italiano, il Catanese si colloca a metà tra classicismo e tardo romanticismo e rispetto alla generazione successiva “il più lontano dal Rapisardi è, di fatto il D'Annunzio, il meno lontano è il Pascoli, a cui fu rimproverata la posa di «fanciullo» come al Rapisardi quella di titano" (Vaccalluzzo, 1930). La sua produzione mostra "una notevole discrasia fra la forma legata al classicismo, ora elegante ora paludata, e il contenuto di sostegno a moderne istanze sociali, quali scienza, progresso, libertà" (Ciccia, 1997, p. 4). La critica polacca lo accosta ai poeti della generazione di Carducci, ovvero Cavallotti, Costanzo, Panzacchi e soprattutto Cesareo e Guerrini. E anche la critica italiana avvicina Rapisardi a diversi autori come Zanella, Bertacchi e Guerrini. Proprio quest'ultimo, uno dei detrattori, è l'autore più spesso accostato a Rapisardi e tale riscontro è presente anche nel giudizio della Gurgul.

Al di là delle polemiche accademiche col Carducci e le accuse di anticlericalismo, esiste una gran mole di scritti ${ }^{31}$ di Rapisardi ancora tutta da scoprire. Si spera che questo invito alla riscoperta del Poeta possa portare a nuove traduzioni e a nuovi studi comparatistici, contribuendo a leggere il vate etnèo al di là dei meri aspetti filosofici e polemici.

Concludendo, dalla critica polacca Rapisardi è quasi sconosciuto e il suo nome è quasi sempre citato o associato a Il Lucifero. Non sono emerse testimonianze critiche sufficienti per capire in che modo gli studiosi polacchi fossero venuti a conoscenza del Catanese. Il presente

31 Rapisardi ha scritto moltissimo; il poeta stesso ha riveduto la propria intera bibliografia, pubblicata dall'editore catanese Giannotta in sei poderosi volumi dal 1894 al 1897, per i quali si rimanda alla bibliografia in calce. 
articolo non esaurisce il discorso su Rapisardi, soprattutto per quanto riguarda le motivazioni che hanno spinto critici e traduttori a occuparsi di lui. Rimangono ancora diverse domande aperte e l'articolo fornisce degli strumenti bibliografici che potranno essere utili per un ulteriore e più ampio studio. Non si esclude infatti che un controllo più approfondito e sistematico delle riviste letterarie e culturali del primo dopoguerra possa portare alla scoperta di nuove fonti e traduzioni dimenticate, dando così la possibilità a italianisti polacchi e traduttologi di approfondire il discorso.

\section{BIBLIOGRAFIA}

Asor Rosa, A. (1966² [1965]). Scrittori e popolo. Il populismo nella letteratura italiana contemporanea. Vol. I. Roma: Samonà e Savelli.

Bąbiak, G. P. (Ed.). (2014). ,,Ludzie osobni” polskiej kultury. Korespondencja Jana Lorentowicza i Zenona Przesmyckiego-Miriama z lat 1899-1938. Warszawa: Wydział Polonistyki Uniwersytetu Warszawskiego.

Barbagallo, S. (1999). Il caso Rapisardi: La verità sulla polemica col Carducci. Paternò: Gazzetta dell'Etna.

Campanozzi, A. (Ed.). (1899). Onoranze a Mario Rapisardi. Catania: Di Mattei.

Cassara, A. (1962). Mario Rapisardi nel cinquantenario della morte. Sicvlorvm Gymnasivm, gennaio-giugno.

Centorbi, G. (1975). Mario Rapisardi "poeta imbalsamato". In V. Consoli, \& S. Nicolosi (Eds.), Immagini di Catania (pp. 236-240). Catania: Ites.

Cesareo, G. A. (1912). M. Rapisardi. Fanfulla della Domenica, 14 gennaio.

Ciccia, C. (1997). Mario Rapisardi poeta e letterato. La gazzetta dell'Etna, 18 luglio, 4.

Colajanni, N. (1894). Avvenimenti di Sicilia e le loro cause. Palermo: Remo Sandron.

D’Agata, M. (1987). I poeti e le poesie in onore di Mario Rapisardi nel $75^{\circ}$ anniversario della scomparsa (1912-1987). Catania: Edizioni della Società Storica Catanese.

De Roberto, F. (1881). Polemica Rapisardi-Carducci. Catania: Giannotta.

Dickstein-Wieleżyńska, J. (1931). Nox. Przegląd Humanistyczny, 2, 28.

Ejsmond, J. (1921). Sonet. Kurier Polski, 19 giugno, 5. 
Falaschi, G. (1979). Luigi Russo. Prose polemiche dal primo al secondo dopoguerra. Milano: Feltrinelli.

Finocchiaro Chimirri, G. (1995). La dimensione catanese nelle riviste letterarie del primo 900. Catania: C.U.E.C.M.

Giarrizzo, G. (1987). Il caso Rapisardi. In S. Zappulla Muscarà (Ed.). (1991), Mario Rapisardi. Atti del convegno nazionale di studi in occasione del XVII Premio Letterario Brancati-Zafferana. Zafferana Etnea, 20-22 dicembre 1987 (pp. 7-25). Catania: Maimone.

Giordano, F. (Ed.). (1998). Mario Rapisardi. Dall'anima inviar l'ultimo sogno. Miscellanea in onore del poeta. Catania: Edizioni della Fenice.

Giordano, F. (2007). Sul "Lucifero" di Mario Rapisardi, nel CXXX anniversario del poema. Catania: La Fenice.

Giordano, F. (Ed.). (2012). Mario Rapisardi. Poesie Religiose. Catania: Boemi.

Girardi, E. N. (2008). Letteratura italiana e religione negli ultimi due secoli. Milano: Jaca Book.

Grasso, A. (2019). Catania. I viali e le piazze. La nuova città del Novecento. Viagrande: Algra.

Guglielmino, S. (1978³ [1971]). Guida al Novecento. Milano: Principato.

Gurgul, M. (2006). Echa włoskie w prasie polskiej (1860-1939). Kraków: Universitas.

Gutowski, W. (2001). Z próżni nieba ku religii życia. Motywy chrześcijańskie w literaturze Młodej Polski. Kraków: Wydawnictwo Literackie.

Heistein, J. (1987). Historia literatury włoskiej. Zarys. Wrocław: Zakład Narodowy im. Ossolińskich.

Krukowska, H., \& Ławski, J. (Eds.). (2014). Roman Zmorski. Lestaw. Szkic fantastyczny. Białystok: Alter Studio.

La Vecchia, T. (2001). Mario Rapisardi. Un caso umano oltre la poesia. Agorà, VII(Ott.-Dic.), 58-61.

Lange, A. [Wrzesień, A.] (1904). Z współczesnej literatury włoskiej. Bluszcz. Pismo tygodniowe illustrowane dla kobiet, 30, 357-358.

Lipińska, J. (1921). Rozczarowanie. In A. Lange, \& A. Tom, (Eds). Panteon literatury wszechświatowej. Italia, Vol. 24 (p. 249). Warszawa: Polska Składnica Pomocy Szkolnych.

Lubczyńska, A. (2017). Tygodnik Słowa Polskiego. Bezpłatny dodatek niedzielny do Stowa Polskiego poświęcony nauce, literaturze i sztuce (19021903). Rocznik historii prasy polskiej, 2(46), 73-89. 
Manganaro, A. (2012). Mario Rapisardi professore. Archivio Storico per la Sicilia Orientale, 2, 54-60.

Mann, M. (1933). Literatura włoska. In S. Lam (Ed.), Wielka literatura powszechna (pp. 85-254). Vol. II. Warszawa: Trzaska, Ewert, Michalski.

Marrené-Morzkowska, W. (2014). Grupa poetów z 1840 roku. Roman Zmorski. In H. Krukowska, \& J. Lawski (Eds.), Roman Zmorski, Lesław. Szkic fantastyczny (pp. 348-363). Białystok: Alter Studio. Reprinted from: Przegląd Tygodniowy Życia Społecznego, Literatury i Sztuk Pięknych, $1880,1-4$.

Miszalska, J., Gurgul, M., Surma-Gawłowska, M., \& Woźniak, M. (Eds.). (2007). Od Dantego do Fo. Włoska poezja i dramat w Polsce (od XVI do XXI). Kraków: Collegium Columbinum.

Niceforo, N. (1872). Mario Rapisardi: Studio Critico-letterario. Catania: Rizzo.

Nicolosi, G. (1921). Mors et Vita di Mario Rapisardi. Recensione critica con prefazione di G. Battiati. Catania: Stab. Tipografico del Popolo.

Patanè, G. (1946). Il prometeo etneo. In G. Patanè, Sicilia amorosa (pp. 63-138). Milano: Valsecchi.

Pelczar, J. S. (19144 [1909]). Masonerya. Jej istota, zasady, dążności, poczatki, rozwój, organizacja, ceremoniat i dziatanie. Wedtug pewnych przeważnie masońskich źródet. Kraków: Skład Główny w Księgarni G. Gebethera i Spółki.

Porębowicz, E. (1933). Literatura włoska. In S. Lam (Ed.), Panteon wielkich twórców poezji i prozy. Antologia literatury powszechnej (pp. 673-754), Vol. I. Warszawa: Trzaska, Ewert, Michalski.

Przesmycki, Z. (1901). Poezja. Chimera, 2(4-5), 318-325.

Puzyna, J. (1921). Góra fatalna. In A. Lange, \& A. Tom (Eds.), Panteon literatury wszechświatowej. Italia, Vol. 24 (p. 249). Warszawa: Polska Składnica Pomocy Szkolnych.

Rapisardi, M. (1887). Le poesie religiose. Catania: Tropea.

Rapisardi, M. (1888). Versi di Mario Rapisardi, scelti e riveduti da esso. Milano: Ulisse Lombardi e c.

Rapisardi, M. (1895). Le poesie religiose. Nuova edizione accresciuta. Catania: Giannotta.

Rapisardi, M. (1894-1897). Opere di Mario Rapisardi, ordinate e corrette da esso. Voll. I-VI. Catania: Giannotta.

Rygier, M. (1902). Z współczesnej literatury włoskiej. Tygodnik Słowa Polskiego, 22, 2-3. 
Russo, L. (1967). La critica letteraria contemporanea. Firenze: Sansoni. Salwa, P. (1997). Historia literatury włoskiej. Warszawa: Semper.

Samperisi, G. (1922). La poesia di Mario Rapisardi. Palermo: Ant. Trimarchi. Santangelo, L. (2012). Mario Rapisardi, Catania ignora il centenario. "Una persona prima che un viale". Meridionews, 4 gennaio. Retrieved from https://catania.meridionews.it/articolo/6839/mario-rapisardi-cataniaignora-il-centenario-una-persona-prima-che-un-vialel.

Sapegno, N. (1969, [Edizione italiana 1948]). Historia literatury włoskiej w zarysie (Z. Matuszewicz, \& K. Kasprzyk, Trans.). Warszawa: PWN.

Scuderi, E. (1968). (Ed.). Mario Rapisardi. Antologia poetica. Catania: Giannotta.

Święcicki, J. A. (1880). Przegląd najnowszej literatury włoskiej. Biblioteka Warszawska. Pismo poświęcone naukom, sztukom i przemysłowi, 2(6), 365-400.

Tomaselli, A. (Ed.). (1915). Mario Rapisardi. Pensieri e giudizi, con l'aggiunta delle Odi Civili e degli aforismi di L. A. Seneca e P. Siro. Palermo: G. Pedone Lauriel.

Tomaselli, A. (1932). Commentario rapisardiano con numerose lettere di illustri scrittori a Mario Rapisardi. Catania: Etna.

Treccani (n.d). Rygier, Maria Anna. Retrieved from https://www.treccani.it/ enciclopedia/maria-anna-rygier_(Dizionario-Biografico).

Tylusińska-Kowalska, A.(2011). Literacki pejzaż Sycylii: Leonardo Sciascia, Gesualdo Bufalino, Vincenzo Consolo, Luisa Adorno, Matteo Collura. Warszawa: DiG.

Ugniewska, J. (1985). Historia literatury wtoskiej XX wieku. Warszawa: PWN. Ugniewska, J. (Ed.). (2001). Historia literatury włoskiej XX wieku. Warszawa: PWN.

Vaccalluzzo, N. (1930). Rapisardi. Un poeta della Natura e del Mistero. Palermo: Remo Sandron. Retrieved from http://rapiasrdi.altervista.org/ critica_a_rapisardi.htm.

Verdirame, R. (2012). La Polonia nelle pagine risorgimentali siciliane. Kwartalnik Neofilologiczny, 59(2), 175-182.

Vigo-Fazio, L. (1913). Oblìo e odio alla memoria di Mario Rapisardi (Intervista con Amelia Poniatowski, compagna del Poeta). Il Tirso, 16 novembre, 1 .

Vigo-Fazio, L. (Ed.). (1930). Mario Rapisardi. Prose Poesie e lettere. Torino: Formica. 
Waszkiewicz, W. M. (2017). Wojsko szatana, wojsko Niepokalanej. Polonia Christiana. https://www.pch24.pl/wojsko-szatana--wojsko-niepokalanej,48544,i.html\#ixzz6cZbAkaoO.

Wędkiewicz, S. (1930). Faszyzm a kultura intelektualna Włoch powojennych. Przegląd Wspótczesny, 34, 308-362.

Wilczak, M. (2016). Julia Dickstein-Wieleżyńska (1881-1943). Monografia dokumentacyjna. Warszawa: IBL.

Zanolla, V. (1993). Un poeta lasciato nell'oblìo. Historia, 37(425), 86-93.

Zanolla, V. (Ed.). (2011). Mario Rapisardi dall'ombra alla luce. Antologia poetica. Pavia: Medea.

Żaboklicki, K. (2008). Historia literatury włoskiej. Warszawa: PWN.

Riassunto: Questo articolo vuole costituire un primo approccio verso uno studio critico, comparatistico e bibliografico della dimenticata figura del catanese Mario Rapisardi (1844-1912), "il vate etneo". Poeta e polemista, egli si espresse nella temperie del Positivismo durante il periodo risorgimentale e post-risorgimentale italiano distinguendosi per la sua tempra etica che non gli fece dismettere la tensione prometeica verso gli ideali. Per essi il poeta entrò in contrasto sia col mondo accademico sia con quello ecclesiastico con il conseguente isolamento letterario, ma non sociale e umano tanto che al suo funerale presero parte oltre centomila persone. Il presente articolo è anche un invito alla riscoperta e alla traduzione in polacco dell'opera del Rapisardi - pressoché inesistente visto che risultano tradotte in polacco solo quattro brevi poesie: tre pubblicate nel 1921 (Disinganno, La montagna fatale, Dinanzi a un ritratto) e una nel 1931 (Nox). Le traduzioni sono rispettivamente di Jadwiga Lipińska, Józef Puzyna, Julian Ejsmond e Julia Dickstein-Wieleżyńska. L'articolo ripercorre cronologicamente la critica polacca su Rapisardi, a partire dal 1880 con gli interventi della scrittrice Waleria Marrené-Morzkowska e del traduttore Julian Adolf Święcicki, fino ai tempi odierni, e si basa sui contributi di studiosi di letteratura come Maurycy Mann, nonché su articoli apparsi su riviste letterarie come Przegląd Humanistyczny e Przegląd Wspótczesny.

Parole chiave: Rapisardi, Catania, Polonia, traduzioni, poesia 